\title{
Avaliação da presença de extensões anteriores dos seios maxilares por meio de tomografia computadorizada por feixe cônico
}

\section{Evaluating the presence of previous extensions of maxillary sinuses through cone beam computed tomography}

Artigo

Original

\author{
Roberta Mansur Caetano ${ }^{1}$ \\ Jairo Conde Jogaib ${ }^{2}$ \\ Alcemar Gasparini Netto ${ }^{3}$ \\ Carlos Dias de Oliveira ${ }^{4}$ \\ José Luiz Cintra Junqueira ${ }^{5}$ \\ Márcio Yara Buscatti ${ }^{6}$
}

\author{
Original \\ Paper \\ Recebido em \\ $09 / 2010$ \\ Aprovado em \\ $12 / 2011$
}

Palavras-chave:

Resumo:

Seio maxilar

Tomografia computadorizada por feixe cônico

Extensão anterior
O terço médio da face comporta os seios paranasais, e entre eles os seios maxilares são os maiores. Eles estão presentes ao nascimento, crescem até a puberdade até que todos os dentes permanentes tenham irrompido, por processo fisiológico conhecido como pneumatização. Podem apresentar extensões para o rebordo alveolar, região anterior, tuberosidade da maxila, palato, osso zigomático e região orbitária. A formação de septos ósseos, transversais, sagitais e oblíquos, dentro do seio é frequentemente notada. A tomografia computadorizada por feixe cônico proporciona uma avaliação precisa da localização dos seios maxilares, importante no planejamento cirúrgico para instalação de implantes dentários. Esse estudo teve como objetivo avaliar a prevalência da extensão anterior dos seios maxilares, em pacientes de ambos os gêneros e diferentes faixas etárias, por meio da tomografia computadorizada por feixe cônico. A amostra foi composta por 200 tomografias, 116 pertencentes ao gênero feminino e 84 ao masculino, totalizando 400 seios maxilares avaliados. O critério escolhido para classificar como extensão anterior, considerou os seios maxilares que se estendiam da face distal do canino em direção à linha média. 12,5\% apresentaram extensão anterior (50 seios), 96\% estendendo até caninos (48 seios) e 4\% até incisivos laterais ( 2 seios). 66\% dos pacientes com extensão anterior, ela se apresentava bilateralmente. Não houve correlação com o gênero, lado e idade.

\section{Abstract}

The midface involves the paranasal sinuses, which the maxillary sinuses are the largest ones. They are present at birth, grow till puberty until all the permanent teeth have erupted by a physiological process known as pneumatization. They can present extension to the alveolar ridge, previous region, maxillary tuberosity, palate, zygomatic bone and orbital region. The formation of transverse, sagittal and oblique bony septa inside the sinus is often noted. The cone beam computed tomography provides an accurate assessment of the location of the
Key words:

\section{Maxillary sinus}

Cone beam computed tomography

previous extension

\footnotetext{
${ }^{1}$ Prof. assistente das Disciplinas Imaginologia I e II do Centro Universitário de Volta Redonda - UniFOA; Mestranda em Radiologia e Imaginologia \& Diagnóstico Estomatológico na Faculdade São Leopoldo Mandic - Campinas

${ }^{2}$ Doutor em Radiologia; Prof. Responsável das Disciplinas Imaginologia I e II do Centro Universitário de Volta Redonda - UniFOA; Vice-Presidente do Centro Universitário de Volta Redonda - UniFOA

${ }^{3}$ Especialista em Radiologia e Estomatologia; Mestrando em Ciências da Saúde e Meio Ambiente; Prof. assistente das Disciplinas Imaginologia I e II, Estomatologia e Semiologia do Centro Universitário de Volta Redonda - UniFOA

${ }^{4}$ Especialista em Radiologia; Prof. assistente das Disciplinas Imaginologia I e II, Estomatologia do Centro Universitário de Volta Redonda - UniFOA

${ }^{5}$ Doutor em Radiologia e em Ortodontia; Diretor geral da Faculdade São Leopoldo Mandic

${ }^{6}$ Doutor em Radiologia
} 
maxillary sinus, it is important in surgical planning for dental implant placement. This study aimed to evaluate the prevalence of previous extension of the maxillary sinuses in patients of both genders and different age groups, by means of cone beam computed tomography. The sample consisted of 200 scans, 116 belonging to the female and 84 male, performing a total of 400 maxillary sinuses. The criterion chosen to classify as previous extension considered the maxillary sinuses that extended from the canine distal towards the midline. $12.5 \%$ had previous extension (50 sinuses), $96 \%$ extending to canines (48 sinuses) and $4 \%$ lateral incisors (2 sinuses), $66 \%$ of patients with previous extension presented bilaterally. There was no correlation with gender, side and age.

\section{Introdução}

O seio maxilar é o maior dos seios paranasais, apresenta-se como uma cavidade preenchida por ar, que se comunica com a fossa nasal através do óstio sinusal maxilar. Varia em relação à forma e ao tamanho, em indivíduos diferentes, podendo apresentar variações entre os lados direito e esquerdo, em um mesmo indivíduo. O seio maxilar pode ocupar toda a maxila com expansões em todas as direções, segundo a literatura. A formação de septos, completos e incompletos, dentro do seio é frequentemente notada.

A necessidade de exames mais completos em informações na área maxilofacial, fez com que nos valêssemos do uso da tomografia computadorizada por feixe cônico (TCFC) que nos possibilita estudo mais acurado. A imagem da estrutura óssea pela TCFC pode ser analisada por completo, sem sobreposição e sem magnificação, com visão tridimensional do rebordo alveolar, permitindo a observação dos limites anatômicos do seio maxilar, na avaliação prévia aos procedimentos cirúrgicos, que é de suma importância para o planejamento e execução do tratamento adequado para cada paciente.

Todo o assoalho do seio maxilar merece atenção diante dos procedimentos reabilitadores utilizando implantes ósseointegrados, entretanto, poucos trabalhos são encontrados na literatura com relação às extensões anteriores dos seios maxilares, o que nos motivou a realizar a presente pesquisa.

\section{Revisão da Literatura}

De acordo com Navarro, Navarro, Navarro (2003) o crescimento dos seios paranasais é lento durante o período fetal, mas, após o nascimento, acelera-se em estirões de crescimento, que ocorrem na infância, puberdade e adolescência.

Sharan, Madjar (2008) concluiram que a pneumatização é um processo fisiológico que ocorre em todos os seios paranasais durante $\mathrm{o}$ período de crescimento, levando-os a aumentar em volume, sendo o seio maxilar o maior, que inicia seu desenvolvimento em dez semanas no útero. Depois do nascimento, ele continua seu crescimento com o desenvolvimento do rebordo alveolar, com os dentes entrando em oclusão. Aos 12/ 13 anos, o assoalho do seio está no nível do assoalho nasal e aos 20 anos com a conclusão da erupção dos terceiros molares, a pneumatização do seio termina e ele atinge cinco milímetros abaixo do assoalho nasal. Em estudo com 152 casos, os autores concluíram que, após extração de dentes posteriores, ocorre o fenômeno conhecido como a quarta expansão, que foi comprovado com radiografias panorâmicas.

Miniti, Bento, Butugan (2000) afirmaram que o seio maxilar ou antromaxilar é uma cavidade no interior do osso maxilar, que tem comunicação com a fossa nasal através do óstio, situado acima e atrás no meato médio. A parede inferior ou soalho tem relação com a abóbada palatina e com os alvéolos dentários. As raízes dos molares e pré-molares podem 
formar saliências no soalho do seio, do qual estão separadas apenas por uma delgada lâmina óssea e, às vezes, esta lâmina pode estar ausente. A parede superior, ou teto, é representada pelo soalho da órbita, onde encontra-se o canal do nervo infraorbitário. A parede posterior está em relação com a fossa pterigopalatina e possui importante relação de vizinhança com a parede externa das fossas nasais.

Em um trabalho realizado por Kim et al. (2003), o limite anterior do seio maxilar, foi na região do $1^{\circ}$ pré-molar, em $58 \%$ dos casos (14 lados), 33\% na região de caninos (8 lados) e $8 \%$ na região de $2^{\circ}$ pré-molar ( 2 lados). $\mathrm{O}$ limite posterior foi na área da tuberosidade maxilar e $3^{\circ}$ molar em $94 \%$ dos casos e $6 \%$ na região de $2^{\circ}$ molar.

Segundo Arieta et al. (2005) em relação à forma dos seios maxilares encontramos extensões para o rebordo alveolar, região anterior, tuberosidade da maxila, palato duro, osso zigomático e região orbitária. Os autores utilizaram uma amostra composta por 100 exames contendo radiografias periapicais e avaliaram a prevalência de extensões anteriores, que foi correspondente a $12 \%$ e desses $42 \%$ mostraram-se bilaterais. $\mathrm{O}$ critério para classificação das extensões anteriores foram seios maxilares estendendo-se da face distal do canino em direção à linha média.

Lee, Lee, Kim (2010) avaliaram 236 seios maxilares em 204 pacientes, com exclusão de casos com alterações patológicas, utilizando tomografia computadorizada, quanto à situação e prevalência de septos no seio maxilar. Foram encontrados septos em $24,6 \%$ dos 236 seios maxilares, com $27,3 \%$ localizados na região anterior, $50 \%$ na região média e 22,7\% na região posterior.

Hauman, Chandler, Tong (2002) afirmaram que a estreita relação anatômica do seio maxilar com as raízes dos molares, pré-molares e em alguns casos, os caninos superiores, podem levar a várias complicações endodônticas e periodontite periapical, resultando em sinusite maxilar de origem dentária. $\mathrm{O}$ advento da implantodontia, o edentulismo maxilar e a necessidade de colocação de implantes de titânio na proximidade ou interior do seio maxilar, levaram ao desenvolvimento da técnica amplamente conhecida como levantamento do seio maxilar, foi o que relataram Maia et al. (2007).

Nimigean et al. (2008) realizaram estudo em 50 esqueletos secos, 30 dentados e 20 edêntulos e concluíram que o assoalho dos seios maxilares representa uma área de perigo para a implantologia oral.

Rodrigues, Vitral (2007) descreveram as principais técnicas tomográficas existentes e suas aplicações na Odontologia. Os autores ressaltaram que resultaram em maior acurácia no diagnóstico e se tornou o exame de eleição para imagens do tecido ósseo do complexo maxilomandibular, com inúmeras indicações, dentre elas avaliação dos seios paranasais e avaliação dos leitos alveolares para implantes dentários. Maestre-Ferrin et al. (2010) observaram que a prevalência de septos é de 13 a $35,3 \%$ dos seios maxilares e para evitar complicações durante a elevação do seio, um meticuloso estudo da cavidade é necessário, de preferência por tomografia computadorizada, pois a radiografia panorâmica demonstrou ter baixa sensibilidade e especificidade na identificação dessas estruturas.

\section{Materiais e Métodos}

O material de estudo constitui-se dos arquivos digitais de 200 exames de tomografia computadorizada por feixe cônico, efetuados em pacientes encaminhados com solicitação do referido exame ao Serviço de Documentação Odontológica - SDO, da cidade de Volta Redonda, estado do Rio de Janeiro, no período de julho de 2009 a dezembro de 2010. Após aprovação pelo Comitê de Ética em Pesquisa da Faculdade São Leopoldo Mandic, a amostra foi estudada quanto à prevalência da extensão anterior do seio maxilar, segundo gênero e faixa etária. Foram utilizados pacientes de ambos os gêneros, com intervalo etário de 13 a 84 anos.

O critério escolhido para classificação das extensões anteriores baseou-se no trabalho de Arieta et al. (2005), que considerou os seios maxilares que se estendiam da face distal do canino em direção à linha média (Figuras 1 e 2).

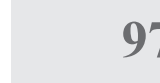

\section{(1)

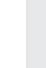 \\ (1)} 要 


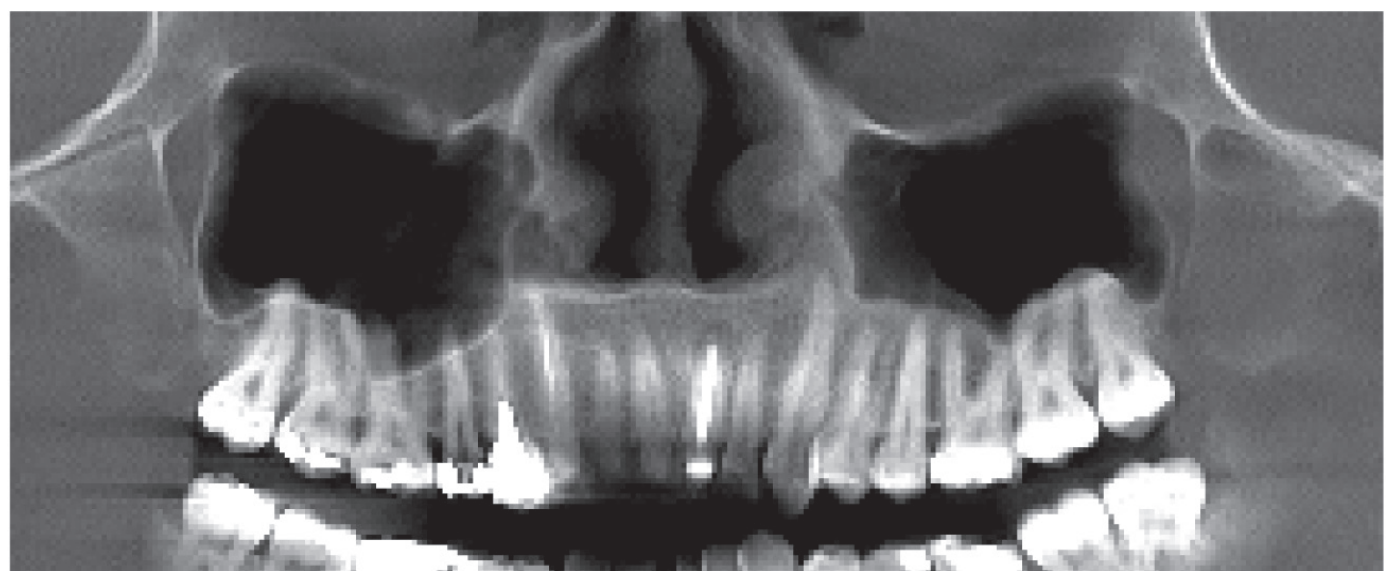

Figura 1- Seio maxilar com limite anterior na região dos primeiros pré-molares, bilateralmente- Reconstrução multiplanar panorâmica

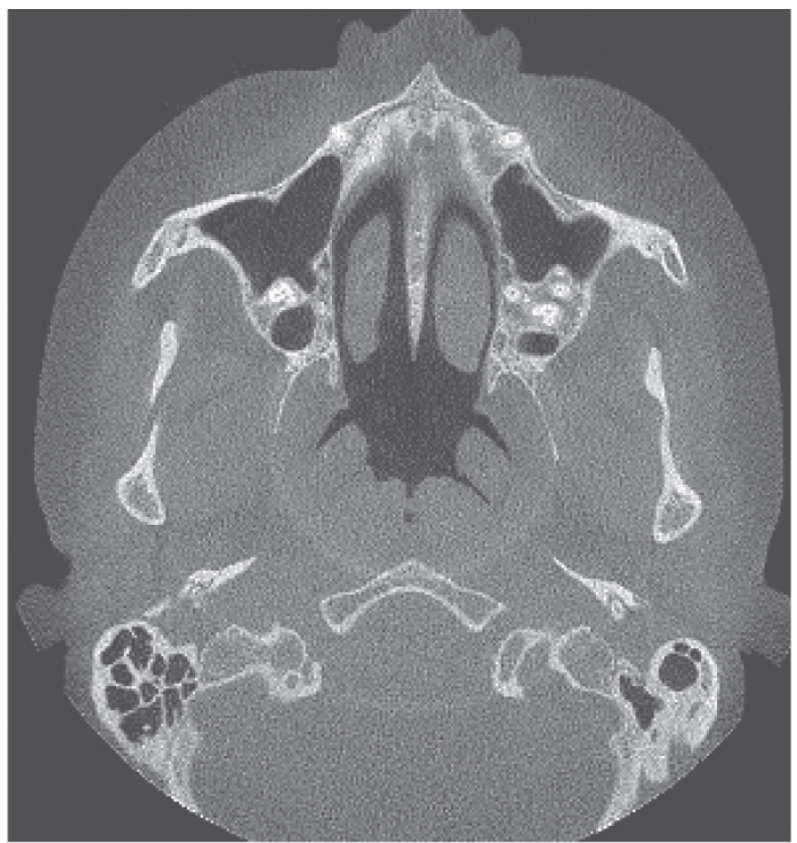

Figura 2- Seio maxilar com limite anterior na região dos primeiros pré-molares, bilateralmente- Reconstrução multiplanar axial

\section{Resultados}

$\mathrm{Na}$ amostra em estudo, a extensão anterior do seio foi verificada em 30 dos 200 exames tomográficos avaliados, sendo assim, a prevalência da extensão anterior do seio maxilar foi de $15 \%$
(Tabela 1). O teste de qui-quadrado revelou que não houve diferença significativa $(\chi 2=0,3155 ; \mathrm{p}$ $=0,5743)$ quanto às prevalências observadas para o gênero masculino $(16,7 \%)$ e feminino $(13,8 \%)$.

Tabela 1 - Distribuição de frequências da presença de extensão anterior do seio maxilar, segundo gênero.

\begin{tabular}{|c|c|c|c|c|c|c|}
\hline \multirow{2}{*}{ Gênero } & \multicolumn{2}{|c|}{ Presença } & \multicolumn{2}{c|}{ Ausência } & \multicolumn{2}{c|}{ Total } \\
\cline { 2 - 7 } & $\mathbf{f}_{\mathbf{i}}$ & $\mathbf{F}_{\mathbf{r} \%}$ & $\mathbf{f}_{\mathbf{i}}$ & $\mathbf{f}_{\mathbf{r} \%}$ & $\mathbf{f}_{\mathbf{i}}$ & $\mathbf{f}_{\mathbf{r} \%}$ \\
\hline Masculino & 14 & 16,7 & 70 & 83,3 & 84 & 100,0 \\
\hline Feminino & 16 & 13,8 & 100 & 86,2 & 116 & 100,0 \\
\hline Total & 30 & 15,0 & 170 & 85,0 & 200 & 100,0 \\
\hline
\end{tabular}


Considerando-se a faixa etária (Tabela 2), não houve diferença significativa entre as prevalências da extensão anterior do seio maxilar, apesar do valor de $p$ ter sido limítrofe $(\chi 2=13,3 ; p=0,0651)$.

Tabela 2 - Distribuição de frequências da presença de extensão anterior do seio maxilar, segundo faixa etária.

\begin{tabular}{|c|c|c|c|c|c|c|}
\hline \multirow{2}{*}{$\begin{array}{c}\text { Faixa } \\
\text { etária }\end{array}$} & \multicolumn{2}{|c|}{ Presença } & \multicolumn{2}{c|}{ Ausência } & \multicolumn{2}{c|}{ Total } \\
\cline { 2 - 7 } & $\mathbf{f}_{\mathbf{i}}$ & $\mathbf{F}_{\mathbf{r} \%}$ & $\mathbf{f}_{\mathbf{i}}$ & $\mathbf{f}_{\mathbf{r} \%}$ & $\mathbf{f}_{\mathbf{i}}$ & $\mathbf{f}_{\mathbf{r} \%}$ \\
\hline $\mathbf{1 3}-\mathbf{2 1}$ & 4 & 21,1 & 15 & 78,9 & 19 & 9,5 \\
\hline $\mathbf{2 2}-\mathbf{3 0}$ & 1 & 20,0 & 4 & 80,0 & 5 & 2,5 \\
\hline $\mathbf{3 1}-\mathbf{3 9}$ & 8 & 36,4 & 14 & 63,6 & 22 & 11,0 \\
\hline $\mathbf{4 0}-\mathbf{4 8}$ & 5 & 12,2 & 36 & 87,8 & 41 & 20,5 \\
\hline $\mathbf{4 9 - 5 7}$ & 6 & 13,3 & 39 & 86,7 & 45 & 22,5 \\
\hline $\mathbf{5 8}-\mathbf{6 6}$ & 6 & 14,0 & 37 & 86,0 & 43 & 21,5 \\
\hline $\mathbf{6 7}-\mathbf{7 5}$ & 0 & 0,0 & 19 & 100,0 & 19 & 9,5 \\
\hline $\mathbf{7 6}-\mathbf{8 4}$ & 0 & 0,0 & 6 & 100,0 & 6 & 3,0 \\
\hline Total & 30 & 15,0 & 170 & 85,0 & 200 & 100,0 \\
\hline
\end{tabular}

Em 38,8\% (155) dos 400 seios maxilares avaliados nos exames tomográficos, o limite anterior dos mesmos localizava-se na região dos primeiros pré-molares, em 37,3\% (149) dos seios, situava-se na região dos segundos prémolares, em $11,5 \%$ (46) na região de primeiros molares, em 12,0\% (48) na região de caninos e em $0,5 \%$ (2) em incisivos laterais (Figura 3).

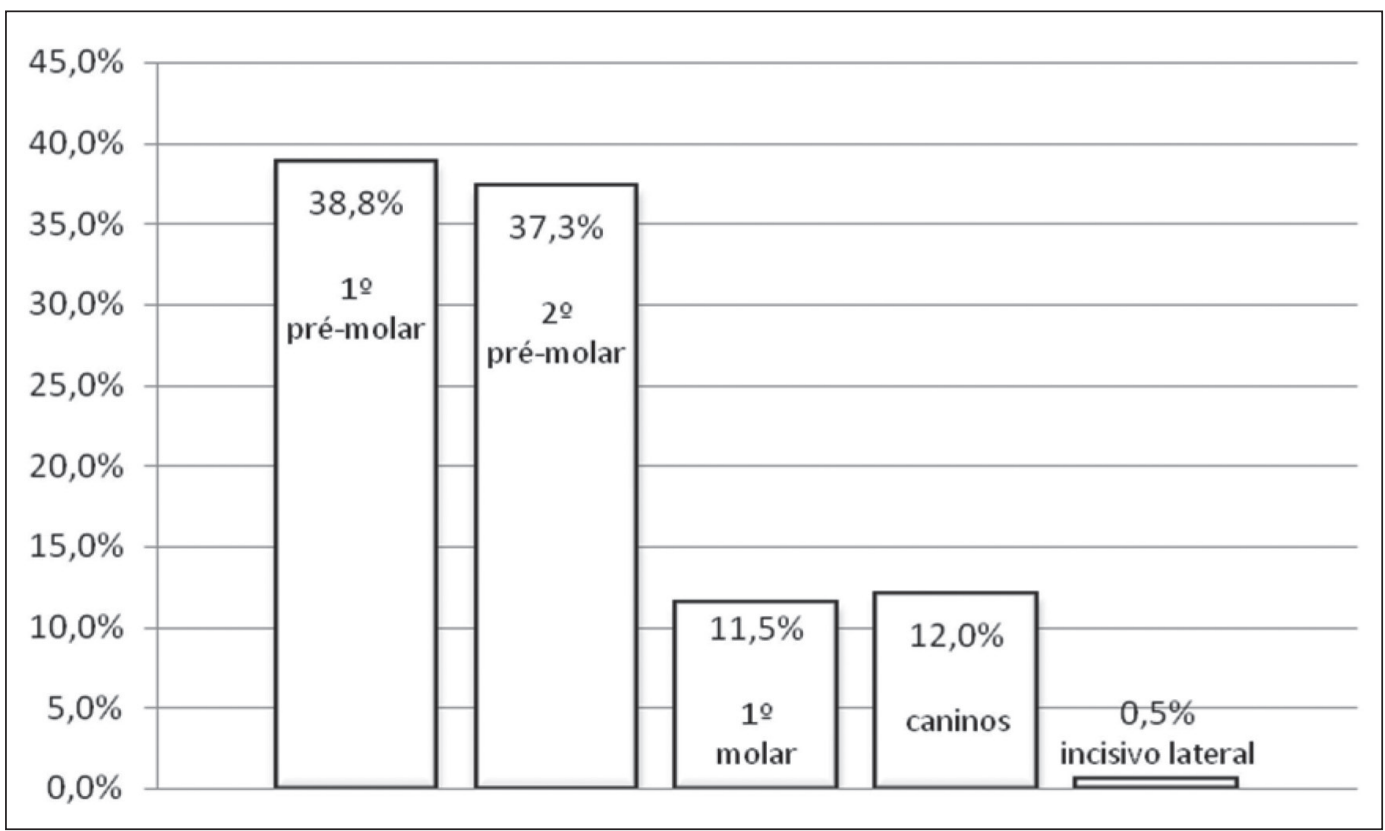

Figura 3- Diagrama de barras da localização do limite anterior do seio maxilar.

Dos 50 seios maxilares com extensão anterior, $96 \%$ o limite anterior se localizava na região de caninos e $4,0 \%$ na região de incisivos laterais. 


\section{Discussão}

Utilizando como referência o trabalho de Arieta et al. (2005) para classificação das extensões anteriores dos seios maxilares, no presente estudo, a extensão anterior do seio foi verificada em 30 dos 200 exames tomográficos avaliados, portanto, a prevalência foi de $15 \%$, acima dos valores encontrados no trabalho de Arieta et al. (2005) que foi de $12 \%$, contradizendo Hauman, Chandler. Tong (2002) afirmaram que, em raros casos, se estende até a região da raiz do canino. Portanto, dos 400 seios maxilares avaliados, 50 apresentavam extensão anterior, sendo que, em 48 casos a extensão foi até caninos e 2 até incisivos laterais.

Dos pacientes que apresentavam extensão anterior (30 pacientes), $66 \%$ eram bilaterais (20 pacientes), bem acima dos valores encontrados por Arieta et al. (2005) que foi de $42 \%$.

Não foi observada correlação da extensão anterior do seio maxilar com o gênero, com prevalência nos exames de $13,8 \%$ no feminino e $16,7 \%$ no masculino. Não houve correlação com os lados direito e esquerdo e também não houve correlação com a idade.

O limite anterior do seio maxilar, do total de 400 avaliados, foi de $38,8 \%$ em $1^{\circ}$ pré-molar, $37,3 \%$ em $2^{\circ}$ pré-molar, $11,5 \%$ em $1^{\circ}$ molar, $12 \%$ em canino e $0,5 \%$ em incisivo lateral. A prevalência maior foi em $1^{\circ}$ prémolar como na pesquisa de Kim et al (2003), que foi de $58 \%$ dos casos, porém, os outros resultados foram bem diferentes, encontraram $33 \%$ na região de caninos e $8 \%$ na região de $2^{\circ}$ pré-molar.

\section{Conclusão}

A prevalência da extensão anterior do seio maxilar avaliada por tomografia computadorizada por feixe cônico foi de $15 \%$ dos exames. Dos seios maxilares com extensão anterior, $96 \%$ se estendiam a região de caninos e $4 \%$ a região de incisivos laterais. Em $66 \%$ dos casos a extensão anterior ocorreu bilateralmente. Não houve correlação com o gênero, nem com a idade. 
1. Arieta LC, Silva MAA, Rockenbach MIB, Veeck EB. Extensões dos seios maxilares detectadas em radiografias periapicais. Revista Odonto Ciência - Fac. Odonto/ PUCRS. 2005 jan-mar; 20(47): 18-22.

2. Hauman CHJ, Chandler NP, Tong DC. Endodontic implications of the maxillary sinus: a review. International Endodontic Journal. 2002; 35: 127-141.

3. Kim HJ, Yoon HR, Kim KD, Kang MK, KwakHH,ParkHDetal.Personal-computerbased three-dimensional reconstruction and simulation of maxillary sinus. Surg Radiol Anat. 2002; 24:393-399.

4. Lee WJ, Lee SJ, Kim HS. Analysis of location and prevalence of maxillary sinus septa. J Periodontal Implant Sci. 2010; 40:56-60.

5. Maestre-Ferrin L, Galán-Gil S, RubioSerrano M, Peñarocha-Diago M, Peñarocha-Oltra D. Maxillary sinus septa: A systematic review. Med Oral Patol Oral Cir Bucal. 2010 mar; 15(2):e383-e386.

6. Maia Filho ALM, Teixeira ERL, Araújo KS, Santos IMSP, Leal NS. Seio maxilar e sua relação de proximidade com as raízes dos dentes superiores posteriores: Uma revisão bibliográfica. XI Encontro Latino Americano de Iniciação Científica e VII Encontro Latino Americano de PósGraduação - Universidade do Vale do Paraíba; 2007: 1929-1932.

7. Miniti A, Bento RF, Butugan $\mathrm{O}$. Otorrinolaringologia: Clínica e Cirúrgica. $2^{a}$ ed. São Paulo: Atheneu; 2000. p. 11-28.
8. Navarro JAC, Navarro PL, Navarro ML. Anatomia da cavidade nasal e seios paranasais. In: Campos CAH, Costa HOO. Tratado de Otorrinolaringologia. Volume I- Fundamentos. São Paulo: Roca; 2003. p. 592-610.

9. Nimigean V, Nimigean VR, Mãru N, Sãlãvãstru DI, Bãditã D, Tuculina MJ. The maxillary sinus floor in the oral implantology. Romanian Journal of Morphology and Embrilogy. 2008; 49(4): 485-489.

10. Ritter L, Lutz J, Neugebauer J, Scheer M, Dreiseidler T, Zinser MJ, et al. Prevalence of pathologic findings in the maxillary sinus in cone-beam computerized tomography. Oral Surg Oral Med Oral Pathol Oral Radiol Endod. 2011; 111: 634-640.

11. Rodrigues AF, Vitral RWF. Aplicações da tomografia computadorizada na Odontologia. Pesq Bras Odontopediatria Clin Integr. 2007 set-dez; 7(3): 317-324.

12. Sharan A, Madjar D. Maxillary sinus pneumatization following extractions: A radiographic study. Int J Oral Maxillofac Implants. 2008; 23:48-56.

13. Silveira VM, Netto BA, Côsso MG, Fonseca LC. A utilização da tomografia computadorizada na avaliação da comunicação bucosinusal. Arq Bras Odontol. 2008; 4(1): 24-27.

14. Tiwana OS, Kushner GM, Haug RH. Maxillary sinus augmentation. The Dental Clinics of N Am. 2006; 50: 409-424.

\section{Endereço para Correspondência:}

Roberta Mansur Caetano

robmansur@hotmail.com

Rua 40, nº 20 , Vila Santa Cecília

Volta Redonda - RJ

CEP: 27.260-200

Informações bibliográficas:

Conforme a NBR 6023:2002 da Associação Brasileira de Normas Técnicas (ABNT), este texto científico publicado em periódico eletrônico deve ser citado da seguinte forma: CAETANO, Roberta Mansur; JOGAIB, Jairo Conde; NETTO, Alcemar Gasparini; OLIVEIRA, Carlos Dias de; JUNQUEIRA, José Luiz Cintra; BUSCATTI, Márcio Yara. Avaliação da presença de extensões anteriores dos seios maxilares por meio de tomografia computadorizada por feixe cônico. Cadernos UniFOA. Volta Redonda, Ano VI, n. 17, dezembro 2011. Disponivel em: <http://www.unifoa.edu.br/cadernos/edicao/17/95.pdf> 